\title{
Surround Suppression Sharpens the Priority Map in the Lateral Intraparietal Area
}

\author{
Annegret L. Falkner, ${ }^{1,5 *}$ B. Suresh Krishna, ${ }^{1,5,6 *}$ and Michael E. Goldberg ${ }^{1,2,3,4,5}$ \\ ${ }^{1}$ Mahoney Center for Brain and Behavior, Department of Neuroscience, and Departments of ${ }^{2}$ Neurology, ${ }^{3}$ Psychiatry, and ${ }^{4}$ Ophthalmology, Columbia \\ University College of Physicians and Surgeons, and ${ }^{5}$ New York State Psychiatric Institute, New York, New York 10032, and ${ }^{6}$ Bernstein Center for \\ Computational Neuroscience and German Primate Center, Goettingen, Germany 37077
}

In the visual world, stimuli compete with each other for allocation of the brain's limited processing resources. Computational models routinely invoke wide-ranging mutually suppressive interactions in spatial priority maps to implement active competition for attentional and saccadic allocation, but such suppressive interactions have not been physiologically described, and their existence is controversial. Much evidence implicates the lateral intraparietal area as a candidate priority map in the macaque (Macaca mulatta). Here, we demonstrate that the responses of neurons in the lateral intraparietal area (LIP) to a task-irrelevant distractor are strongly suppressed when the monkey plans saccades to locations outside their receptive fields. Suppression can be evoked both by flashed visual stimuli and by a memorized saccade plan. The suppressive surrounds of LIP neurons are spatially tuned and wide ranging. Increasing the monkey's motivation enhances target- distractor discriminability by enhancing both distractor suppression and the saccade goal representation; these changes are accompanied by correlated improvements in behavioral performance.

\section{Introduction}

In the visual world, stimuli are in constant competition for allocation of the brain's limited processing resources. Salient stimuli are often not relevant to the task at hand, but can nevertheless transiently capture attention and result in disadvantageous behaviors (Egeth and Yantis, 1997; Bisley and Goldberg, 2003b; Peck et al., 2009). For example, a jungle predator may have difficulty monitoring his elusive prey when there is a distracting insect flying in his field of view. It would be disadvantageous for this predator to lose focus on the location of the prey, either through an eye movement or a shift of attention to the location of the insect. Human subjects can reduce the capture of attention by an abruptly appearing, task-irrelevant stimulus by attending to a different, task-relevant location before the distractor appears (Egeth and Yantis, 1997). This reduction in attentional capture could be achieved in the brain by enhancing the neural activity encoding the location of the task-relevant stimulus or by suppressing the neural activity associated with the location of the task-irrelevant stimulus. In principle, an efficient neural strategy

\footnotetext{
Received May 6, 2010; revised July 19, 2010; accepted July 22, 2010.

M.E.G. was supported by National Eye Institute Grants R24EY015634, R01EY01497, and R01EY017039, and the Kavli, Keck, Dana, and Zegar Foundations. A.L.F. was supported by the National Science Foundation Graduate Research Fellowship and the Ruth L. Kirschstein National Research Service Award. B.S.K. was supported by the German Ministry for Education and Science Grant BMBF 01GQ0433 to the Bernstein Center for Computational Neuroscience, and by a Gatsby Foundation award. We are grateful to Drs. Mo Osman, Moshe Shalev, and Girma Asfaw for veterinary care, Yana Pavlova for veterinary assistance, John Caban for machining, Glen Duncan for electronics, and Latoya Palmer for facilitating everything.

${ }^{*}$ A.L.F. and B.S.K. contributed equally.

Correspondence should be addressed to either of the following: Annegret L. Falkner, 1051 Riverside Drive, Unit 87, New York, NY 10027, E-mail: alf2111@columbia.edu; or B. Suresh Krishna, German Primate Center, 4 Kellnerweg, 37077 Goettingen, Germany, E-mail: skrishna@dpz.eu.

DOI:10.1523/JNEUROSCI.2327-10.2010

Copyright $\odot 2010$ the authors $\quad 0270-6474 / 10 / 3012787-11 \$ 15.00 / 0$
}

might incorporate both mechanisms simultaneously, though how such interactions occur is not yet understood.

Many models of attentional and saccadic processing posit that the allocation of visual attention and the selection of saccade targets are both based on the dynamically evolving peak of activity in a map-like representation of spatial priority (Schall, 1995; Gold and Shadlen, 2000; Itti and Koch, 2000; Fecteau and Munoz, 2006; Goldberg et al., 2006; Serences and Yantis, 2007; Armstrong et al., 2009). Current evidence suggests that such priority-map representations exist in several interconnected brain regions, including the lateral intraparietal area (LIP), the frontal eye field (FEF), and the superior colliculus (SC) (Keller and McPeek, 2002; Thompson and Bichot, 2005; Goldberg et al., 2006). It is commonly theorized that different spatial locations on this map mutually suppress each other over large distances to facilitate the evolution of a clear peak of activity that can serve as the focus of visual attention and select the target for saccadic eye movements (Koch and Ullman, 1985; Itti and Koch, 2001; Deco et al., 2002; Constantinidis and Wang, 2004). Such mutual suppression is also considered crucial for maintaining a localized and persistent focus of attention that is resistant to abruptly appearing distractors (Constantinidis and Wang, 2004; Wong et al., 2007), and for the programming of sequential saccades (Xing and Andersen, 2000). Long-ranging interactions across the priority map may also be necessary for the global computations of relative reward value that are known to affect the priority map in LIP (Dorris and Glimcher, 2004; Sugrue et al., 2004).

Despite this theoretical interest, suppression has never been explicitly studied in LIP (or any other priority map area), and its very existence is controversial. Surround suppression has been postulated to explain the decrease in activity with increasing set size in a visual search task (Balan et al., 2008), but it has also been 
A
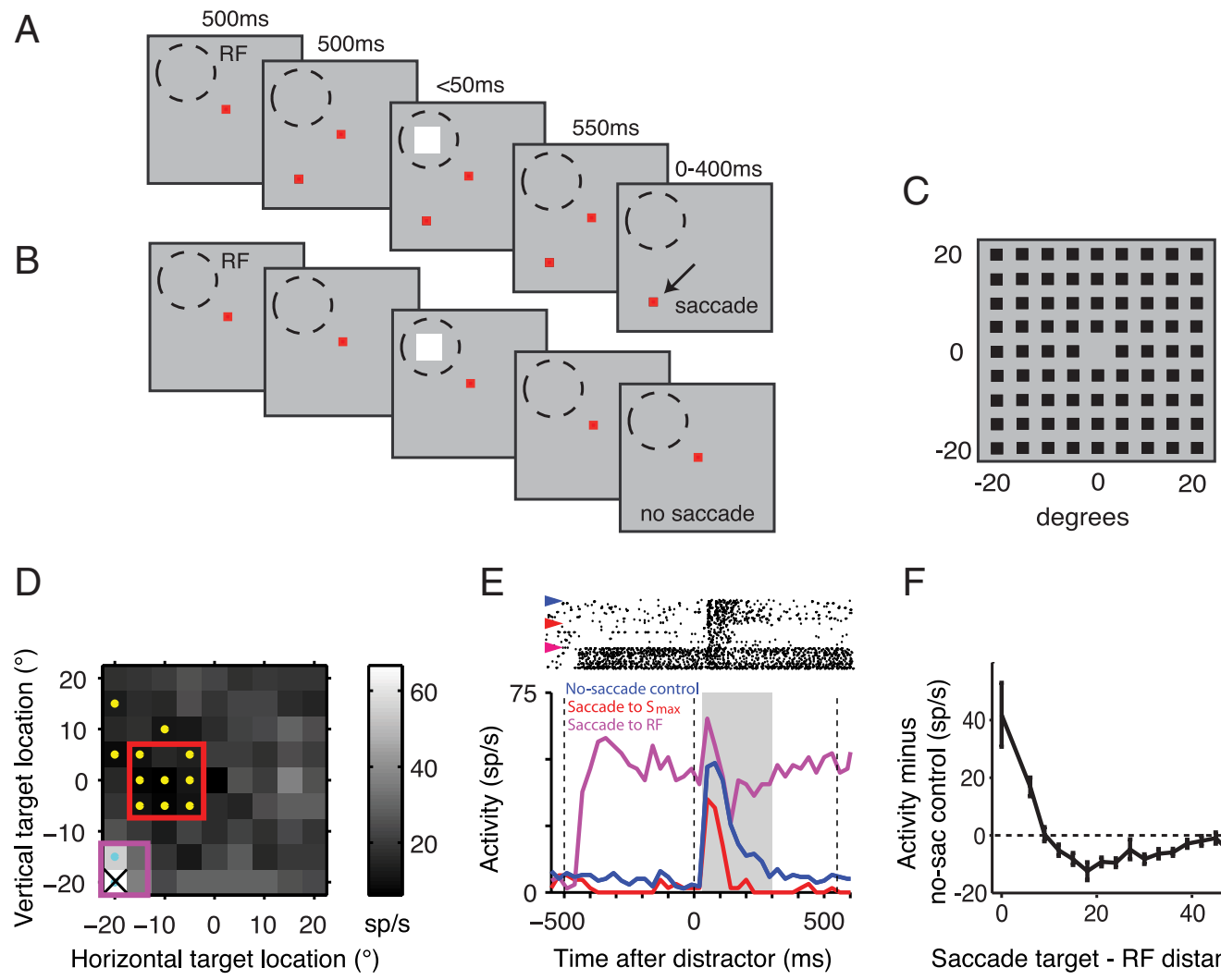

$\mathrm{F}$

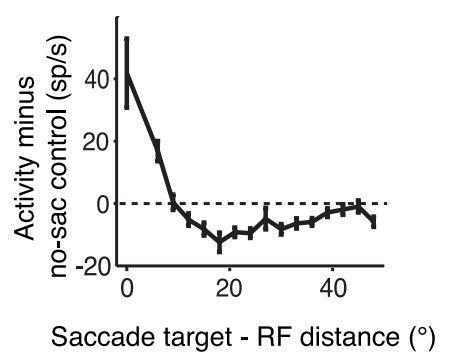

Figure 1. Task design. A, Target-mapping task: monkeys fixated for $500 \mathrm{~ms}$, after which a target appeared at a location randomly chosen from a $40 \times 40^{\circ}$ grid of locations in the visual field, with $5^{\circ}$ spacing in most cases ( $($ ). After $500 \mathrm{~ms}$, a distractor was flashed briefly ( $<50 \mathrm{~ms}$ ) at the RF center. After a $550 \mathrm{~ms}$ delay, the fixation spot was turned off, and the monkey made a saccade to the target to obtain the reward. $\boldsymbol{B}$, The no-saccade control task was otherwise identical, except that the fixation spot was never turned off, and monkeys maintained fixation throughout the trial to obtain the reward. C, Grid of potential target locations. D, Suppression of example LIP neuron by a saccade plan to the surround. Grayscale map of response to the distractor (average firing rate from 30 to $300 \mathrm{~ms}$ after distractor onset) as a function of saccade target location with respect to the central fixation point. Target locations with yellow/cyan dots are significantly suppressed (yellow) or enhanced (cyan) relative to the no-saccade control ( $p<0.05$, $t$ test with Bonferroni correction for 80 simultaneous comparisons). Boxed locations indicate saccade target locations defined as the $\mathrm{RF}$ (magenta) and $S_{\max }($ red) for the rasters and PSTHs in $\boldsymbol{E}$. X at $-20,20$ indicates distractor location. $\boldsymbol{E}$, PSTHs and rasters from the example neuron, aligned to distractor onset: saccade plan to the $S_{\max }$ leads to a reduced distractor response (red) compared to both the no-saccade control response (blue) and the response when saccade is planned to the RF (magenta). Rasters are sorted by trial type. Dashed vertical lines indicate time of saccade target onset (left), distractor onset (middle), and time of saccade go-cue (right). $\boldsymbol{F}$, Distractor response varies with distance of saccade target from the RF center. An example neuron's distractor response during the target-mapping task (minus the response during the no-saccade control, horizontal dashed line at 0 ) is plotted as a function of the distance between the saccade target and the RF center. Error bars show SEMs ( $n=$ number of locations contributing to each data point).

recently argued that LIP has no surround suppression (Churchland et al., 2008). In this study, we demonstrate that the priority map in LIP is, in fact, powerfully influenced by surround suppression. Surround suppression in LIP possesses novel properties that have not been demonstrated before in other visual areas.

\section{Materials and Methods}

We used three male rhesus monkeys (Macaca mulatta) weighing 8-12 kg in this experiment. All experimental protocols were approved by the Animal Care and Use Committees at Columbia University and the New York State Psychiatric Institute, and complied with the guidelines established by the Public Health Service Guide for the Care and Use of Laboratory Animals. We located the intraparietal sulcus in each monkey using a T1 volume scan obtained on a GE Signa 1.5 T magnet. Using standard sterile surgical techniques and endotracheal isoflurane general anesthesia, we made a $2 \mathrm{~cm}$ trephine hole over the intraparietal sulcus and implanted 12-16 titanium screws in the monkey's skull and used them to anchor an acrylic cap in which we placed a head-holding device, the recording chamber, and the plug for subconjunctival search coils for eye position recording. We used three recording cylinders: (monkey D, left hemisphere; monkey I, right hemisphere; monkey Z, right hemisphere).

Data collection and task design. We used the REX/MEX/VEX system developed at the National Eye Institute's Laboratory for Sensorimotor Research for behavioral control, visual stimulus display and data collection using Dell Optiplex PCs running QNX (REX and MEX) and Win- dows 2000 (VEX). The monkeys sat in a dimly illuminated room with their head fixed and viewed a screen that stood $75 \mathrm{~cm}$ away. Visual stimuli were back-projected onto the screen using a LCD projector (Hitachi CP-X275) with a refresh rate of $75 \mathrm{~Hz}$. We used a photodiode to register the actual times for stimulus onsets and offsets. Fixation point and saccade target stimuli were $0.3^{\circ}$-wide colored squares and distractors were $1.5^{\circ}$-wide white squares. We introduced the electrodes through a guide tube positioned in a $1 \mathrm{~mm}$ grid (Crist Instrument). We recorded single units from area LIP with glassinsulated tungsten electrodes (Alpha Omega Engineering) while the monkeys performed a passive fixation task as white spots flashed sequentially at different locations in the visual field. We amplified, filtered, and discriminated action potentials using an amplitude window discriminator (MEX software). Only well isolated single neurons were studied.

We considered neurons to be in LIP if they showed consistent visual, delay-period, and/or saccade-related response during the memoryguided saccade task or were located between such neurons in that electrode penetration. Of 98 neurons tested, 52 (53.06\%) responded significantly more during the delay period of a memory-guided delayed saccade task ( $t$ test, one-tailed $p<0.05$, average of 49.84 trials per neuron) than at baseline. Every neuron responded to the abrupt onset of a visual stimulus in its receptive field (RF).

Task details. For each neuron we isolated, we identified the center of the RF using flashed spots at $400 \mathrm{~ms}$ intervals (4 per trial, located on a $40 \times 40^{\circ}$ grid with $5^{\circ}$ spacing, $<50 \mathrm{~ms}$ duration) during passive fixation (Fig. 1C). We defined the center of the RF as the spatial location of the 
flashed spot that elicited the maximum activity. We then ran a nosaccade control, where the monkeys fixated a central red fixation spot for $2050 \mathrm{~ms}$. One thousand milliseconds after the monkey achieved fixation, a brief white spot flashed for $<50 \mathrm{~ms}$ ( $2-3$ video frames in $90 \%$ of trials; 1 video frame on the remaining trials) in the center of the RF. The duration of the distractor was independent of all other task parameters, including stimulus locations, timing, and reward size. The no-saccade control was identical to the target-mapping task (below), except that no saccade target appeared and the monkey had to maintain fixation throughout the trial to get his reward. After $\sim 50$ trials of the no-saccade control task, we had the monkey perform several variants of the delayed saccade paradigm. We ran each task only on a subset of neurons, depending on the neuron's isolation quality and the monkey's satiety.

The target-mapping task began with the appearance of a central red fixation spot; $500 \mathrm{~ms}$ after the monkey fixated the central spot, a saccade target appeared at a location randomly chosen from 80 possibilities (on a $40 \times 40^{\circ}$ grid with $5^{\circ}$ spacing). Occasionally, for some neurons, we sampled from a slightly different set of locations, varying either the sampling or the spatial extent of the grid to more closely sample particular regions of space or to ensure sufficient data collection within the limited recording time available. A distractor flashed briefly for $<50 \mathrm{~ms}$ (two or three video frames in $\sim 90 \%$ of trials) at the center of the RF $500 \mathrm{~ms}$ after the saccade target appeared. When the center of the RF coincided with one of the 80 target locations, the distractor flashed on top of the (much smaller) saccade target. The fixation spot disappeared $550 \mathrm{~ms}$ after distractor onset; this was the cue for the monkey to make the saccade (gocue). Monkeys had to keep their eyes within a $3 \times 3^{\circ}$ window until the cue to make the saccade appeared. The mean SD of eye position during the fixation period was $<0.25^{\circ}$ for all sessions. Monkeys had $400 \mathrm{~ms}$ after the go-cue to make a saccade to a $4.5 \times 4.5^{\circ}$ window around the saccade goal. If the monkey's eye was in the window for $100 \mathrm{~ms}$ from 400 to $500 \mathrm{~ms}$ after the go-cue, he received a drop of water or juice as reward. In $<5 \%$ of randomly interleaved trials on this task, no saccade target appeared and the monkey was rewarded for maintaining fixation throughout the trial (interleaved no-saccade control). The distractor-mapping task was similar to the target-mapping task, except that the saccade target location was fixed at the RF center while the distractor location was varied over a $40 \times 40^{\circ}$ grid. In another task variant of the target-mapping task, we also had the monkey make a memory-guided saccade to an effective location in the suppressive surround, while the distractor appeared at the RF center ( $\sim 75$ trials). We typically used this task after $\sim 500$ trials on the target-mapping task, after which an effective location could be identified. Finally, we measured neuronal properties on the cued-reward task, where we cued either a large or a small reward randomly on each trial by the saccade target color. For most neurons, we first ran a control task where the saccade target could be one of two different colors, but each was associated with the same reward $(\sim 50$ trials with distractor at $\mathrm{RF}$ center and saccade to suppressive surround, and $\sim 50$ trials with saccade to RF center and distractor in the suppressive surround). Next, the monkeys performed the cued-reward task, where the two chosen saccade target colors was associated with different reward sizes. In the first block, the distractor appeared at the RF center and the saccade was made to the suppressive surround ( $\sim 200$ trials) and in the second block, the saccade was made to the RF center and the distractor appeared in the suppressive surround ( $\sim 50$ trials). Actual reward magnitudes were chosen daily based on the monkey's satiety level and his behavioral sensitivity to the difference in reward sizes. The reward ratio between small and large rewards was $\sim 1: 6$ on average, and ranged from 1:3 to 1:10 for all but two neurons (for which we used higher ratios of 1:20 and 1:50, respectively). The apparently large range of reward ratios is explained by the fact that ratios are very sensitive to changes in the denominator when the denominator is small.

On a given trial, the monkey's eye had to remain within a $4.5 \times 4.5^{\circ}$ window around the saccade goal from 400 to $500 \mathrm{~ms}$ after the go-cue for reward delivery. The monkey's eye usually entered this window with the first saccade after the go-cue: for example, in the cued-reward task, in $96.5 \%$ of trials, the first saccade after the go-cue landed within this window. Counting only saccades at least $1.5^{\circ}$ in amplitude, the monkeys usually made only one (92.9\%) or two (6.8\%) saccades during the period between the go-cue and the reward.

Data analysis. All data analysis programs were written in MATLAB (MathWorks). For the target-mapping task, we verified that a given saccade target location lay outside the RF by comparing the response to the onset of the saccade target to the pretarget response (response $30-230 \mathrm{~ms}$ after target onset compared to response -170 to $30 \mathrm{~ms}$ relative to target onset; $t$ test, one-tailed $p<0.05$ ). This insured that the suppression of the distractor response as a result of the saccade plan could not be simply attributed to response adaptation as the result of an excitatory response to the target. We computed population-averaged peristimulus time histograms (PSTHs) by first obtaining PSTHs from each neuron and then finding the mean of these PSTHs. We used ANOVAs to quantify the spatial properties of the surround. To have a sufficient number of trials for these analyses, we first pooled the saccade target locations into nine $10 \times 10^{\circ}$ clusters before performing the ANOVA. When we computed the $S_{\max }$ (defined as the $10 \times 10^{\circ}$ cluster of saccade targets associated with the minimum distractor response), we found that in 2/72 neurons there were two such clusters that were associated with the minimum distractor response. In these two ambiguous cases, we picked one cluster at random as the $S_{\max }$. In the remaining 70 neurons, our algorithm identified a unique $S_{\max }$ and there was no ambiguity. We defined the angular position of the target with respect to the RF as the angle between a line connecting the saccade target to the RF center and a horizontal line passing through the RF center. We used $t$ tests wherever applicable rather than alternatives like the Wilcoxon signed-rank test or the rank-sum test because we consistently observed that whenever there was an occasional discrepancy between the $t$ test and the alternatives (which do assume that the underlying distribution is symmetrical), it was the $t$ test that agreed with the results of permutation tests based on repeated simulations. For the analyses of proportions and latencies, the underlying distributions were very skewed, and we therefore used a permutation test that compared the $z$-statistic for the paired difference between large- and smallreward trials with the null distribution for the paired difference obtained by 10,000 random shuffles of the two distributions being compared.

Error classification. On error trials, the monkeys usually made one (70.5\%), two $(20.8 \%)$, or three $(5.7 \%)$ saccades from a time beginning $350 \mathrm{~ms}$ after distractor appearance. For categorizing error trials, we first classified each saccade in these trials as (1) target directed (if its endpoint lay within $5^{\circ}$ of the saccade goal), (2) distractor directed (if its endpoint lay within $5^{\circ}$ of the distractor goal), or (3) elsewhere directed (if it was neither target directed nor distractor directed). We then used the following scheme to categorize a given error trial: If there were no target-directed or distractor-directed saccades, it was classified as an elsewhere-directed error trial. If only target-directed saccades were present, it was a target-directed error trial, and similarly, if only distractor-directed saccades were present, it was a distractor-directed error trial. If both target-directed and distractor-directed saccades were present, then we classified the trial as target or distractor directed depending on which saccade was earlier.

In $24.5 \%$ of error trials, REX truncated data collection of the eye position signal before the end of the final saccade, and so the saccade endpoint could not be determined directly; in these cases, we classified saccades whose mean direction was within 0.35 radians $\left(20^{\circ}\right)$ of the line joining the fixation point to the saccade target as target-directed saccades, and saccades that were not target directed and whose mean direction was within 0.35 radians of the line joining the fixation point to the distractor location as distractor-directed saccades.

\section{Results}

We studied the effect of surround suppression on the visual responses of a total of 105 LIP neurons LIP in three monkeys (43 in monkey Z, 26 in monkey D, 36 in monkey I). Our dataset consists of LIP neurons with systematically mapped RFs based on their clear visual response to a briefly flashed spot (see Materials and Methods). We considered neurons to be in LIP if they showed consistent visual, delay-period, and/or saccade-related responses during the memory-guided saccade task or were located between 
such neurons in that electrode penetration. A categorical breakdown of the number of neurons contributing to each task type is shown in supplemental Table 1 (available at www.jneurosci.org as supplemental material).

\section{LIP neurons show clear surround suppression by a planned saccade}

We looked for evidence of surround suppression of LIP neural responses by systematically mapping the effect of a visually guided delayed saccade plan on the response to a task-irrelevant distractor in a subset of 72 neurons. In this task (the targetmapping task, Fig. 1A), the monkey fixated the central fixation point and a small red target appeared at a location chosen randomly on each trial from the grid (Fig. 1C). Five hundred milliseconds later, a large, white, salient but task-irrelevant distractor flashed in the center of the RF for $<50 \mathrm{~ms}$ The fixation point disappeared $550 \mathrm{~ms}$ later, which served as a cue for the monkey to make a saccade to the target within $400 \mathrm{~ms}$ to earn a reward. We compared the neuronal response in the target-mapping task to the response in a control task (the no-saccade control task, Fig. $1 B$ ) run in a separate block, in which the monkey was rewarded for maintaining fixation and no saccade target appeared. The no-saccade control task was otherwise identical to the targetmapping task. We also ran a small proportion of no-saccade control trials interleaved within the target-mapping task: the distractor response in this condition was not significantly different from that in the blocked version (mean difference \pm $\mathrm{SEM}=1.16 \pm 1.73$ spikes $/ \mathrm{s}, t$ test $p=0.5027, n=72$ neurons) (supplemental Fig. 1 $A, B$, available at www.jneurosci.org as supplemental material).

In the target-mapping task, a saccade plan to locations in the visual field outside the RF suppressed the response to the distractor flashed at the RF center relative to the distractor response in the no-saccade control task (Fig. 1). Suppression could be evoked from a range of spatial locations. The degree of suppression varied markedly as a function of saccade target location (example response map in Fig. 1D). To quantify the maximal amount of suppression for each neuron, we identified a $10^{\circ} \times 10^{\circ}$ region outside the RF ( $S_{\max }$, red square in Fig. $1 D$ ) from which the saccade plan maximally suppressed the distractor response. We used a randomly selected two-thirds of trials to define the location of the $S_{\max }$ for each neuron. Locations were considered to be outside the RF if the appearance of the saccade target did not elicit a significant response from the neuron (see Materials and Methods). We then cross-validated, using the remaining one-third of trials to estimate the magnitude of the suppression evoked by the saccade plan to locations within $S_{\max }$. When the monkey planned a saccade to regions within $S_{\max }$, the distractor response was reduced compared to the no-saccade control (see example neuron, Fig. $1 E$ ). This reduction was strongly present in our LIP population: in 66 of 72 neurons (91.7\%), planning a saccade to locations within $S_{\max }$ reduced the neuron's response to the distractor relative to the no-saccade control (Fig. $2 B$ ) (two-sample $t$ test, onetailed $p<0.05, n=$ number of trials in the session). Across the population, the distractor response was strongly reduced compared to the no-saccade control task when planning a saccade to locations within $S_{\max }$ (mean reduction $\pm \mathrm{SEM}=14.14 \pm 1.46$ spikes/s, $p<0.0001, n=72$ neurons). In addition, the net distractor response (calculated by subtracting the pre-distractor firing rate $240 \mathrm{~ms}$ before to $30 \mathrm{~ms}$ after distractor onset) was also significantly suppressed when the monkey planned a saccade to locations within $S_{\max }$ compared to the no-saccade control task (mean reduction $\pm \mathrm{SEM}=8.27 \pm 1.19$ spikes $/ \mathrm{s}, p<0.0001, n=$ 72 neurons).

Suppression emerged in the background activity even before the distractor appeared, beginning $100 \mathrm{~ms}$ after the appearance of the saccade target, and continuing through the trial until the go-cue (Fig. $2 A)$ ( $n=72$ neurons). The pre-distractor baseline response ( -500 to $30 \mathrm{~ms}$ around distractor onset) when the monkey planned a saccade to locations within $S_{\max }$ was significantly reduced compared to the no-saccade control task (mean reduction $\pm \mathrm{SEM}=3.43 \pm 1.15$ spikes $/ \mathrm{s} ; p=0.0037, n=72$ neurons). When we recalculated the $S_{\max }$ based on the predistractor baseline response itself, rather than basing it on the distractor response (supplemental Fig. $1 E, F$, available at www. jneurosci.org as supplemental material), an even larger effect on the pre-distractor baseline response was visible (mean reduction $\pm \mathrm{SEM}=7.09 \pm 1.07$ spikes $/ \mathrm{s}, p<0.0001, n=72$ neurons).

\section{Surround suppression is long ranging and radially symmetric in the population}

Suppressive surrounds from individual neurons in LIP had a wide variety of shapes, similar to reports of surround suppression from lower visual areas (Orban, 2008). The amount of suppression elicited by a saccade target varied significantly with its spatial location in 57/72 neurons (Kruskal-Wallis ANOVA, $p<0.05$ ). Surround suppression extends over long distances: the farthest location from which significant suppression could be elicited lay $>21.2^{\circ}$ from the excitatory receptive field center in all 66 neurons that showed significant surround suppression. The mean farthest location was $35^{\circ} . S_{\max }$ lay further than $10^{\circ}$ from the RF center in $65 / 72$ neurons $(90.3 \%)$.

Although individual neurons showed diverse surround shapes, surround suppression averaged across the population was radially symmetric and showed a systematic dependence upon the distance of the saccade target from the RF center. For each neuron, we plotted the average activity from the targetmapping task for each target location (minus the activity in the no-saccade control task) as a function of the distance between the distractor and target locations (example neuron in Figs. $1 F, 2 C$ ). On average, suppression reached a maximum between $12^{\circ}$ and $35^{\circ}$ from the RF center, and remained significant up to $40^{\circ}$ away from the RF center. A similar distance dependence was seen for the baseline response (supplemental Fig. $2 B$, available at www. jneurosci.org as supplemental material). To evaluate the radial symmetry of the surround around the excitatory receptive field center, we plotted the average activity from the target-mapping task at each location outside the RF (minus the activity in the no-saccade control task) as a function of the angular locations of the target with respect to the RF center (Fig. 2D, black). The population-averaged surround was radially symmetric around the RF center, and showed no significant dependence upon the angular location of the saccade target with respect to the RF center (Kruskal-Wallis ANOVA, $p=0.8732$ ).

Individual neurons, however, did not in general have circularly symmetric surrounds. When we replotted the data in the blue trace in Figure $2 \mathrm{D}$ after rotating the location of $S_{\max }$ so that it always lay at $0^{\circ}$, thereby aligning the locations with the maximum amount of suppression, the average response showed a clear dependence upon angular location of the saccade target with respect to the excitatory receptive field center (gray trace, Kruskal-Wallis ANOVA, $p<0.0001$ ). In accordance with this result, the degree of suppression in individual neurons along a ring passing through $S_{\max }$ (with the RF as center) depended significantly upon the angular location of the saccade target in 33/72 
A

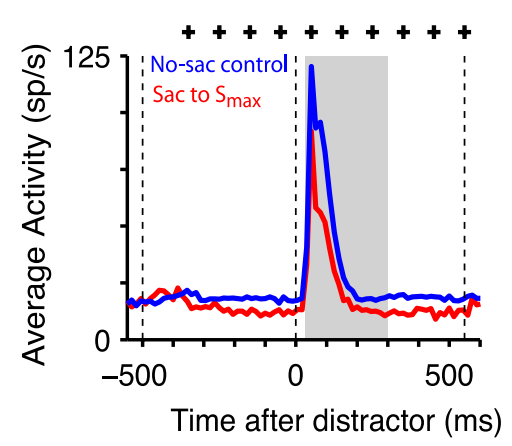

\section{B}

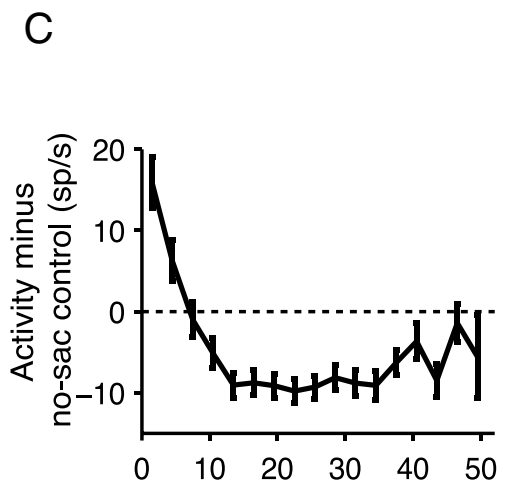

Saccade target - RF distance $\left({ }^{\circ}\right)$
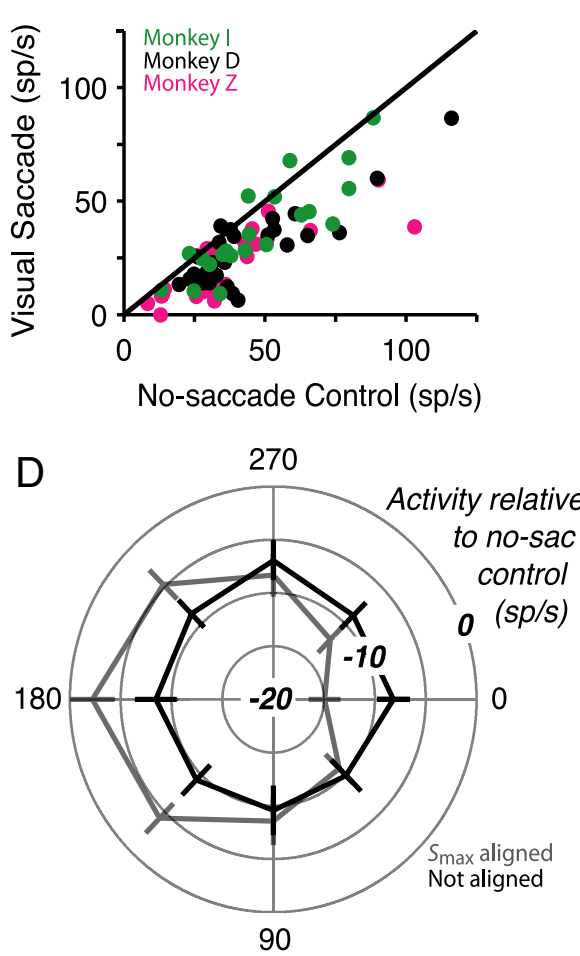

Angle of saccade target relative to $\mathrm{RF}$ location $\left(^{\circ}\right)$

Figure 2. A, Population average PSTH reveals significant suppression of both baseline and distractor response when a saccade is planned to the $S_{\max }(r e d)$ compared to the no-saccade control (blue). Only the one-third of trials that were not used to calculate the $S_{\max }$ contribute to the response in red. Black crosses mark the centers of non-overlapping $100 \mathrm{~ms}$ time bins with a significant difference between responses (paired $t$ test, $p<0.05, n=72$ neurons). $\boldsymbol{B}$, Scatter plot of each neuron's response to the distractor ( 30 -300 ms after distractor onset, gray bar in $A$ ) during the no-saccade control (abscissa) and the saccade plan to $S_{\max }$ condition (ordinate). Paired $t$ test: $p<0.0001, n=72$. Green, black, and magenta circles indicate neurons from monkeys I, D, and Z, respectively. C, Distractor response varies with distance of saccade target from the RF center. Population-averaged distractor response during the target-mapping task (minus the response during the no-saccade control, horizontal dashed line at 0 ) is plotted as a function of the distance between the saccade target and the RF center. Averaged tuning curves were first calculated for each neuron and then averaged together to produce the population average. Error bars show SEMs. $n=$ number of neurons contributing to each data point. $\boldsymbol{D}$, Population-averaged suppression extends in all directions from the RF. A polar plot shows average level of suppression in 8 binned angular directions (bin size $=45^{\circ}$ ) around the RF center. Polar tuning plots were first computed for each neuron and then averaged together. Error bars are as in $\boldsymbol{C}$. The magnitude of suppression (spikes/s) is plotted as a function of absolute direction around the RF center (black), and after rotating all target angles so that the center of $S_{\max }$ lies at $0^{\circ}$ (gray). The four circles in the polar plot lie at $-15,-10,-5$, and 0 spikes/s. See also supplemental Figures 1 and 2 (available at www. jneurosci.org as supplemental material).

neurons (45.8\%, Kruskal-Wallis ANOVA, $p<0.05)$. Finally, though the RF centers were all in the contralateral visual field or along the midline, the locations of the $S_{\max }$ for each cell showed only a small tendency to lie in the contralateral hemifield: $18 / 25$ neurons (72.0\%) in monkey $\mathrm{Z}$ and $15 / 22$ neurons (68.2\%) in monkey $\mathrm{D}$ lay in the contralateral hemifield $(p=0.0455$ and $p=$ 0.1356 , respectively, test of proportions), but only $9 / 19$ (47.4\%) neurons in monkey I did ( $p \approx 1$, test of proportions).

\section{Suppression can be maintained without the presence of the visual target}

LIP neurons show enhanced activity throughout the delay period of the memory-guided delayed saccade task (Gnadt and Andersen, 1988). We tested whether this was also true for surround suppression in a subset of 48 neurons by modifying the target-mapping task. Instead of a visually guided delayed saccade, we asked the monkey to make a memory-guided delayed saccade to a saccade target that flashed briefly for $50 \mathrm{~ms}$ at a single location within the suppressive surround. The task was otherwise identical to the target-mapping task (Figs. $1 \mathrm{~A}, 3 \mathrm{~A})$. We briefly flashed a distractor in the RF center $500 \mathrm{~ms}$ after the saccade target appeared. The fixation point disappeared $550 \mathrm{~ms}$ after the distractor disappeared, and the monkey was rewarded for making a saccade to the spatial location of the vanished stimulus. For 40 of the 48 neurons, the neuronal response to the distractor that appeared during the delay period was significantly suppressed relative to the no-saccade control $(83.3 \%$; two-sample $t$ test, one-tailed $p<0.05$ ) (Fig. 3B). Suppression was strongly significant across the population of sampled neurons (mean reduction \pm SEM $=$ $16.09 \pm 2.33$ spikes/s, $p<0.0001, n=48$ neurons) (Fig. 3C). Suppression by a memory-guided saccade, like suppression by a visually guided saccade, began $100 \mathrm{~ms}$ after the saccade target appeared and continued until the go-cue (Fig. $3 B)(n=48$ neurons).

\section{A distractor in the surround transiently suppresses the response to the saccade target}

In a subset of neurons ( $n=37)$, we looked to see whether a flashed distractor in the surround would have a suppressive effect on the maintained activity during a planned saccade to the excitatory receptive field (Fig. 4A). In this task (the distractor-mapping task), a variant of the target-mapping task, the saccade target always appeared at the RF center, while the spatial location of the distractor (rather than the saccade target) was randomized. The distractor-mapping task was otherwise identical to the target-mapping task. As a control, during a small number of interleaved trials, no distractor appeared during the delay period (the no-distractor control). Again we used half the trials to determine the $10 \times 10^{\circ}$ region where the distractor evoked maximal suppression $\left(\mathrm{DS}_{\max }\right)$ and then used the remaining trials to estimate the effect of a distractor flashed within DS ${ }_{\max }$. A distractor flashed within DSmax caused a significant transient suppression of the maintained delay-period response of the neuron. This effect was significant for two consecutive $100 \mathrm{~ms}$ bins following distractor onset (Fig. $4 B$ for the population average) ( $n=37$ neurons). The response 30-300 ms following the distractor flashed within $S_{\max }$ was significantly lower than the response in the same period in the nodistractor control (Fig. $4 C$ ) (mean decrease \pm SEM $=3.00 \pm 1.07$ spikes/s, $p=0.0075, n=37)$. In addition, the saccadic latency on these trials (where distractor appeared within $\mathrm{DS}_{\max }$ ) was significantly longer compared to the interleaved no-distractor control trials, despite the fact that the distractor appeared $550 \mathrm{~ms}$ before the go signal (Fig. $4 D$ ) (mean increase \pm SEM $=13.07 \pm 2.85 \mathrm{~ms}$, $p<0.0001, n=37$ ), confirming that the distractor had a disrupt- 
A

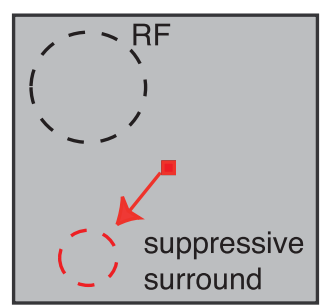

B

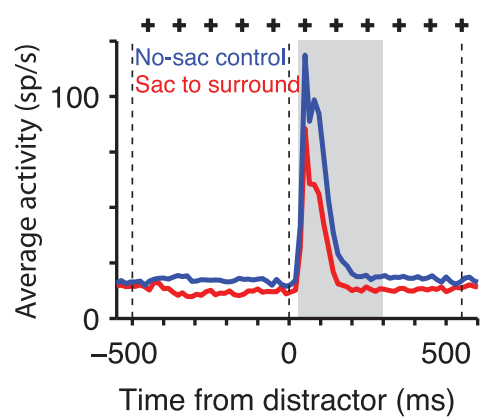

C

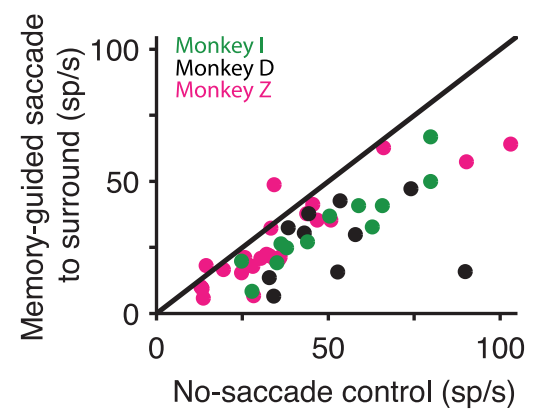

Figure 3. Suppression can be maintained without the presence of the saccade target. $\boldsymbol{A}$, Task design: Identical to the target-mapping task, except thatmonkeys planned a memory-guided saccade to a single fixed location within the surround. The distractor was flashed at the RF center. $\boldsymbol{B}$, Population average PSTH reveals significant suppression of both baseline and distractor response when a memory-guided saccade is planned to the surround (red) compared to the no-saccade control (blue). Bin width $=15 \mathrm{~ms}$. C, Scatter plot of each neuron's response to the distractor (gray bar in $\boldsymbol{B}$ ) during the no-saccade control (abscissa) compared to the saccade plan to the surround (ordinate). One point (control response $=131.70$, saccade plan $=59.64$ ) was omitted for visibility. Paired $t$ test: $p<0.0001, n=48$. The figure format for $B$ and C is otherwise identical to Figure $2, A$ and $B$, respectively.

ing effect on the monkey's saccade plan. Saccade latencies are also longer when averaged across all possible distractor locations (not just the $\mathrm{DS}_{\mathrm{max}}$; mean increase $\pm \mathrm{SEM}=12.35 \pm 3.42 \mathrm{~ms}$, $p<0.0001, n=37$ ) (Fig. 4D).

\section{Increasing expected reward increases the response to the target and decreases the response to the distractor}

We studied the effect of motivation on surround suppression on a subset of neurons in LIP $(n=48)$ using a variant of the targetmapping task. We cued the monkey to expect a large or a small reward using the color of the saccade target to indicate the upcoming reward (cued-reward task). We used two specific spatial configurations (always in separate blocks): to measure the distractor response, we presented the distractor at the RF center (and the saccade target at a fixed location in the suppressive surround), and to measure the target response, we presented the saccade target at the RF center (and the distractor at a fixed location within the suppressive surround). The cued-reward task was otherwise identical to the target-mapping task. We randomly assigned color pairings with large and small rewards during each recording session. Large- and small-reward targets were interleaved within each block of trials. The monkeys learned the reward contingencies associated with the color of the saccade target within a few trials, as indicated by significantly faster saccade latencies and significantly fewer distractor-directed saccades on the large-reward trials than on the small-reward trials (Roesch and Olson, 2003; Hikosaka et al., 2006).

On large-reward trials, the three monkeys never made an error saccade directed toward the distractor. However, on smallreward trials, all three monkeys made a very small, but significant proportion of error saccades directed toward the distractor (permutation $p<0.0001$ in each monkey, mean proportion \pm SEM in monkey: Z, $2.08 \pm 1.07 \%, n=8 ; \mathrm{D}, 1.77 \pm 0.41 \%, n=19 ; \mathrm{I}$, $2.82 \pm 0.76 \%, n=21)$. Monkeys $\mathrm{Z}$ and I also made significantly shorter latency saccades to the saccade targets associated with the large reward than to those associated with the small reward (mean decrease \pm SEM in monkey: $\mathrm{Z}, 83.46 \pm 12.86 \mathrm{~ms}, p=$ $0.0002, n=8 ; \mathrm{D}, 10.21 \pm 7.54 \mathrm{~ms}, p=0.1813, n=19 ; \mathrm{I}, 22.80 \pm$ $9.70 \mathrm{~ms}, p=0.0258, n=21)$. These results indicate that the monkeys could assess the reward value of the trial based on the saccade target color and were more highly motivated during large-reward trials.

The relative magnitude of the cued reward affected the responses to both the distractor (when the monkey planned a sac- cade to the surround and the distractor appeared at the RF center) and the target (when the target and distractor locations were reversed). The neuronal response to the distractor flashed at the RF center on large-reward trials was significantly lower than the response on small-reward trials in three consecutive $100 \mathrm{~ms}$ periods following distractor onset (Fig. $5 A$ ) ( $n=46$ neurons). This reduction was significant in 31 of the 46 neurons taken individually (67.4\%; two-sample $t$ test, one-tailed $p<0.05)$. In the population, the reduced distractor response on large-reward trials was highly significant (Fig. $5 B$ ) (mean decrease \pm SEM $=$ $10.36 \pm 1.83$ spikes $/ \mathrm{s}, p<0.0001, n=46$ neurons).

The neuronal response to the saccade target appearing at the RF center was greater when the monkey expected a large reward instead of a small reward. In the population, the enhanced target response ( $400 \mathrm{~ms}$ before distractor onset to $30 \mathrm{~ms}$ after distractor onset) on large-reward trials was highly significant (Fig. 5C) (mean increase $\pm \mathrm{SEM}=11.15 \pm 2.27$ spikes $/ \mathrm{s}, p<0.0001, n=$ 38 neurons). The target response was significantly higher on large-reward trials in 22 of the 38 neurons taken individually (57.9\%; two-sample $t$ test, one-tailed $p<0.05)$. The enhanced target response, averaged across monkeys, was significant throughout the trial from $100 \mathrm{~ms}$ after saccade target onset until the go-cue (Fig. 5D) ( $n=38$ neurons). The duration of the enhanced target response was variable across monkeys. Monkey D showed an enhanced target response only during the early part of the trial, while the other two monkeys showed an enhanced response throughout the trial until the go-cue (supplemental Fig. $3 C-H$, available at www.jneurosci.org as supplemental material).

To ensure that the effects of varying expected reward were not due simply to a systematic (though unlikely) overlap of the chosen reward associations with LIP color selectivity, we performed a control experiment in a subset of the neurons recorded in the cued-reward task (supplemental Fig. $3 A, B$, available at www. jneurosci.org as supplemental material). Before associating different reward sizes with different colors, we recorded the responses of LIP neurons with both target colors associated with the same reward size, sampling both spatial configurations (distractor at RF center and target in suppressive surround in one block and target at RF center and distractor in suppressive surround in another block). We found no significant behavioral differences ( $n=38$ neurons) between trials with the two saccade target colors in the total percentage of errors (mean difference \pm $\mathrm{SEM}=0.37 \pm 0.67 \%, p=0.58)$, the percentage of distractordirected error saccades (mean difference \pm SEM $=0.036 \pm 0.060 \%$, 
A

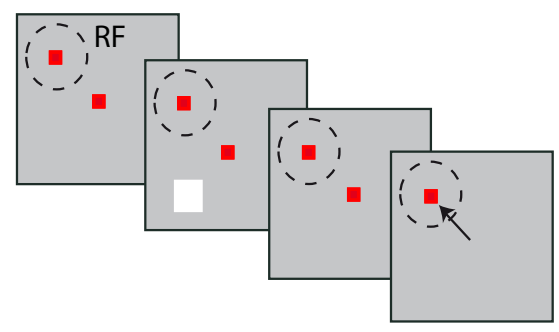

C

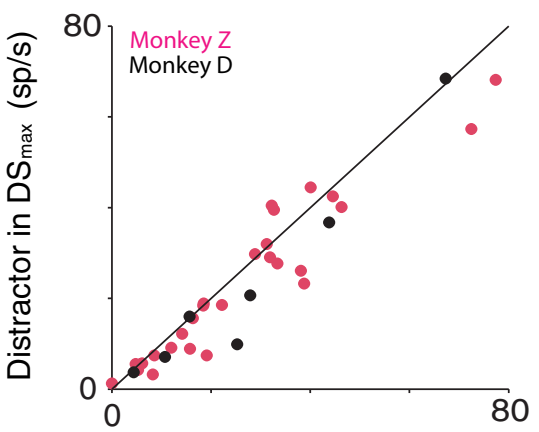

No-distractor control (sp/s)

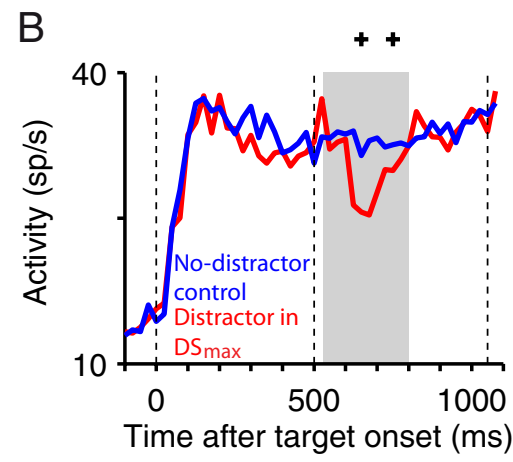

D

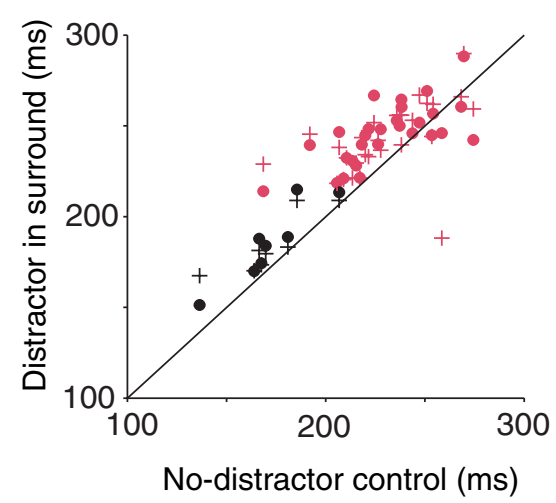

Figure 4. Aflashed distractor elicits suppression.A,Distractor-mapping task:identical to the target-mapping task (Fig. 1A), except that the saccade target location was fixed at the RF center, and the distractor location was chosen randomly on each trial from the $40 \times 40^{\circ}$ grid of locations (Fig. 1C). In interleaved no-distractor controls, the distractor was not flashed. $\boldsymbol{B}$, Distractor onset in the surround evokes transient suppression. Population average PSTHs show the no-distractor control (blue) and the population average response when the distractor was flashed within $D S_{\text {max }}$ calculated from the bottom-up response map (red). Trials used to calculate $D S_{\max }$ not included in the PSTH. PSTHs are aligned to saccade target onset. Black crosses are as in Figure 2A. Bin width $=25 \mathrm{~ms}$. C, Scatter plot of each neuron's

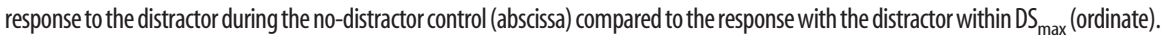
Paired $t$ test: $p=0.007, n=37$. Two points (control response $=176.02,134.35$ and saccade $p l a n=189.92,123.28$ ) were omitted for visibility. D, Distractor appearance leads to slowed saccadic latencies: scatter plot of mean saccadic latency during the no-distractor control (abscissa) versus the mean saccadic latency with the distractor flashed either within DS ${ }_{\max }$ (ordinate, filled circles) or anywhere in the visual field (ordinate, plus signs). Each point represents data from the recording of one neuron. Paired $t$ tests for both comparisons: $p<0.0001$, $n=37$. The figure format for $\boldsymbol{B}$ and $\boldsymbol{C}$ is otherwise identical to Figure $2, A$ and $B$, respectively.

$p=0.50$ ), or saccadic latency (mean difference \pm SEM $=1.44 \pm$ $2.24 \mathrm{~ms}, p=0.53$ ). Before reward association, we also found no significant differences between the two colors in either the distractor response (mean difference $\pm \mathrm{SEM}=0.22 \pm 0.67$ spikes $/ \mathrm{s}$, $p=0.74, n=35$ ) or the target response (mean difference \pm SEM $=2.12 \pm 2.32$ spikes/s, $p=0.36, n=31$ ).

On large-reward trials, the three monkeys never made an error saccade directed toward the distractor. However, on smallreward trials, all three monkeys made a very small but significant proportion of error saccades directed toward the distractor (permutation $p<0.0001$ in each monkey, mean proportion \pm SEM in monkey: Z, $2.08 \pm 1.07 \%, n=8 ; \mathrm{D}, 1.77 \pm 0.41 \%, n=19 ; \mathrm{I}$, $2.82 \pm 0.76 \%, n=21)$. Monkeys $Z$ and I also made significantly shorter latency saccades to the saccade targets associated with the large reward than to those associated with the small reward (mean decrease \pm SEM in monkey: Z, $83.46 \pm 12.86 \mathrm{~ms}, p=$ $0.0002, n=8 ; \mathrm{D}, 10.21 \pm 7.54 \mathrm{~ms}, p=0.1813, n=19 ; \mathrm{I}, 22.80 \pm$ $9.70 \mathrm{~ms}, p=0.0258, n=21$ ). These results indicate that the monkeys could assess the reward value of the trial based on the saccade target color and were more highly motivated during large-reward trials.
Distractor and saccade target responses are correlated with saccade latency We looked for correlations between the neuronal response and the monkeys' saccade latency in the cued-reward task. For small-reward trials, the distractor response was positively correlated with saccade latency on a trial-by-trial basis: increased distractor responses were associated with longer-latency saccades when the distractor was flashed at the RF center. The average correlation coefficient (Spearman's $\rho$ ) between trial-by-trial neuronal activity 30 $300 \mathrm{~ms}$ after distractor onset and saccadic latency was significantly greater than zero (Fig. $5 E)$ (mean \pm SEM $=0.088 \pm 0.024$, $p=0.0006, n=46)$. Conversely, the target response was negatively correlated with saccade latency on a trial-by-trial basis: increased target responses were associated with shorter-latency saccades when the target appeared at the RF center. The average correlation coefficient between trial-by-trial neuronal activity $300-500 \mathrm{~ms}$ after distractor response and saccadic latency was significantly negative (mean \pm SEM $=$ $-0.14 \pm 0.04 \mathrm{~ms} / \mathrm{Hz}, p=0.0005, n=38$ ). In large-reward trials, there was a similar negative correlation coefficient when the target lay at the RF center (mean \pm SEM $=$ $-0.069 \pm 0.027 \mathrm{~ms} / \mathrm{Hz}, p=0.0132, n=$ 38 ), but not when the distractor was flashed at the RF center (mean $\pm \mathrm{SEM}=0.033 \pm$ $0.027 \mathrm{~ms} / \mathrm{Hz}, p=0.2266, n=46)$. Since the reduced distractor response on largereward trials is accompanied by a reduced variance (mean decrease \pm SEM $=8.59 \pm$ $2.43 \mathrm{~Hz}^{2}, p=0.0002, n=46$ ), we interpret this result as reflecting that once distractor responses are highly suppressed (as in the large-reward trials), their reduced residual trial-by-trial variability contributes less to the overall variation in saccadic latency.

\section{The distractor response in LIP predicts erroneous saccades}

The monkeys had an error rate of only $5.0 \%$ on average during large-reward trials. But because they usually made far more errors on small-reward trials (mean error rate $=31.5 \%$ ), we were able to use the small-reward trials to test whether there was any correlation between the distractor response in LIP and the monkey's saccadic behavior. To eliminate any contamination of the distractor response (30-300 ms following distractor onset) from saccade-related signals, we included only error trials where the monkey made an erroneous saccade after $350 \mathrm{~ms}$ following the distractor onset. We divided error trials into three types depending on whether the erroneous saccade was directed toward the distractor (distractor-directed error trials), preemptively toward the target (target-directed error trials), or toward neither the distractor nor the target (elsewhere-directed error trials). The distractor response in LIP was significantly enhanced on distractordirected error trials compared to correct trials (Fig. 6C) (mean increase \pm SEM $=15.80 \pm 3.54$ spikes/s, $p=0.0003, n=18$ neurons). The population average PSTH on distractor-directed 
error trials (Fig. $6 \mathrm{~B}$, red PSTH) was significantly different from the PSTH on correct trials (Fig. $6 \mathrm{~B}$, blue PSTH) from 100 to $300 \mathrm{~ms}$ following distractor onset $(n=$ 18 neurons). The enhancement of the distractor response was specific to distractordirected error trials and was not present for the other two types of error trials. There was no significant difference between the distractor response on elsewhere-directed error trials and correct trials (mean difference $\pm \mathrm{SEM}=2.75 \pm 1.99$ spikes $/ \mathrm{s}, p=$ $0.1691, n=35$ neurons). Similarly, the distractor response on target-directed error trials was actually significantly lower than that on correct trials (mean decrease \pm $\mathrm{SEM}=4.60 \pm 2.48$ spikes $/ \mathrm{s}, p=0.0270$, $n=35$ neurons). The enhanced distractor response therefore predicted the impending erroneous saccade toward the distractor.

There was no significant difference in the target response (from $400 \mathrm{~ms}$ before to $300 \mathrm{~ms}$ after distractor onset) between distractor-directed error trials and elsewhere-directed error trials (mean difference $\pm \mathrm{SEM}=2.29 \pm 3.81$ spikes $/ \mathrm{s}, p=$ $0.5405, n=9$ neurons); we therefore pooled these two types of trials together as error trials with saccades directed away from the target. The target response on these trials (Fig. 6 E, red PSTH) was significantly higher than that on correct trials (Fig. 6E, blue PSTH) (mean increase \pm SEM $=10.03 \pm 1.55$ spikes $/ \mathrm{s}, p<0.0001$, $n=31$ neurons, Fig. $6 F$ ). Thus, a lower target response predicts error trials where the monkey saccades away from the target. On these error trials, a higher distractor response predicts a distractor-directed saccade.

\section{Discussion}

Our results show, for the first time, that LIP neurons have strong suppressive surrounds that influence and sharpen the LIP priority map. By systematically characterizing these effects, we find that suppression of LIP responses is wide-ranging and can therefore link the representations of stimuli that are distant from each other in physical space.

\section{Surround suppression in LIP is affected} by cognitive influences

Surround suppression evoked by visual stimuli is a ubiquitous neural strategy and has been reported in many visual areas, including the retina and lateral geniculate nucleus (Alitto and Usrey, 2008), area V1 (Angelucci and Bressloff, 2006), MT and MST (Allman et al., 1985; Eifuku and Wurtz, 1998; Orban, 2008), and V4 (Desimone et al., 1985, 1993). However the surround suppression we report here in LIP has several unique properties not seen in other visual areas. Most importantly, surround suppression observed in LIP is affected by cognitive processes that are not
B

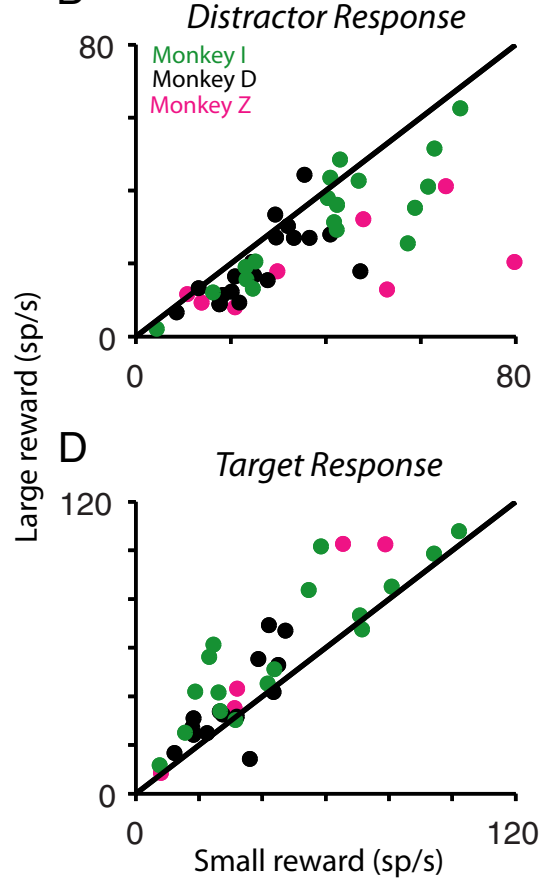

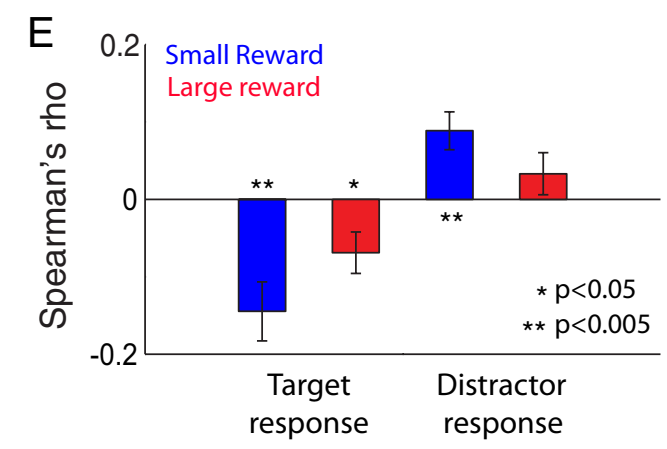

Figure 5. Increasing motivation enhances suppression. $\boldsymbol{A}$, Distractor response is lower during large-reward trials: With distractor in RF and target in the suppressive surround, population average PSTH shows reduced distractor response in large-reward trials (red), compared to small-reward trials (blue). Black crosses are as in Figure 2A. B, Scatter plot of each neuron's response to the distractor during small-reward (abscissa) and large-reward (ordinate) trials. Paired $t$ test: $p<0.0001, n=46$. One point (smallreward: 105.63, large-reward: 79.00) was omitted for visibility. C, Target response is larger during large-reward trials: With target in RF and distractor in the suppressive surround, population average PSTH shows enhanced target response in large-reward trials (red), compared to small-reward trials (blue). Black crosses are as in Figure 2A. D, Scatter plot of each neuron's response to the target ( -400 to $30 \mathrm{~ms}$ after distractor onset, gray bar in C) during small-reward (abscissa) and large-reward (ordinate) trials. Paired $t$ test: $p<0.0001, n=38$. PSTH bin widths: 15 ms. Two points (small-reward: 150.26 and 153.30, large-reward: 184.38, 149.44) were omitted for visibility. The figure format for PSTHs and scatter plots is as in Figure 2. $\boldsymbol{E}$, Correlates of LIP neurons with saccadic behavior in the cued-reward task: Larger LIP neuronal responses are associated with faster saccades to the RF and slower saccades to the surround. In the small-reward condition (blue bars), correlation coefficients were significantly negative (paired $t$ test: $p=0.0005, n=38$ ) with target in RF and distractor in the surround, while slopes were significantly positive with distractor in RF and target in the surround (paired $t$ test: $p=0.0006, n=46$ ). Effect sizes were smaller in the large-reward condition (red bars) and only significant with the target in RF (paired $t$ test: $p=0.0132, n=38$ ) and not with the distractor in the RF (paired $t$ test: $p=0.2266, n=46$ ). See also supplemental Figure 3 (available at www.jneurosci.org as supplemental material).

dependent upon the presence of a particular visual stimulus. We demonstrated this in two ways: first, suppression of LIP responses can be maintained by the memory of a visual stimulus, which will guide a future saccade, whereas the surround suppression demonstrated in lower visual areas was evoked and maintained by a visual stimulus itself. Second, suppression of LIP responses is enhanced when the monkey expects a larger reward as a result of a learned association with a visual stimulus. Increased motivation enhanced the representation of the saccade goal and suppressed the representation of the distractor. The enhancement of the tar- 

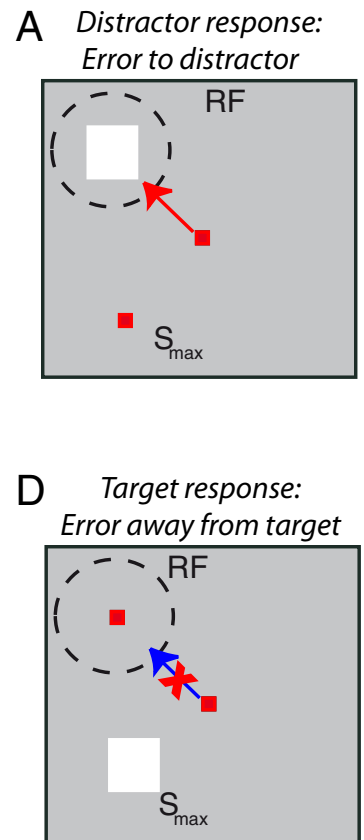

B

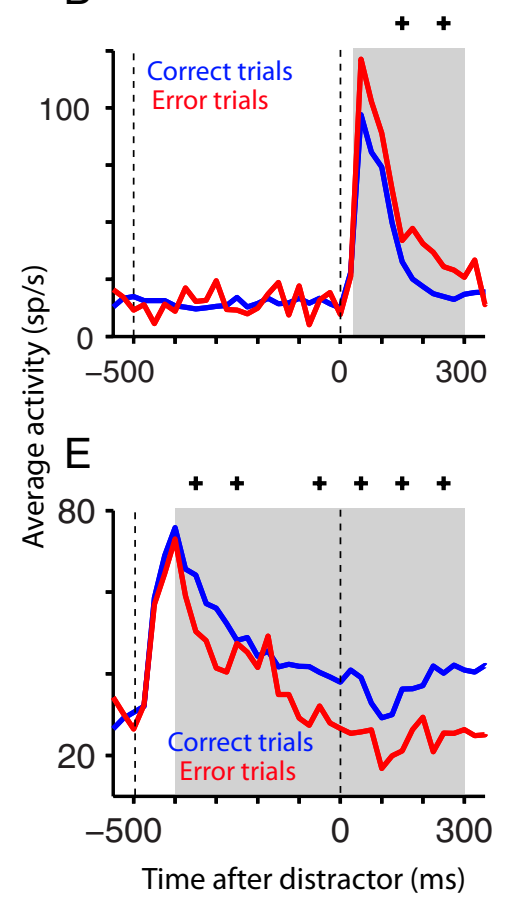

C

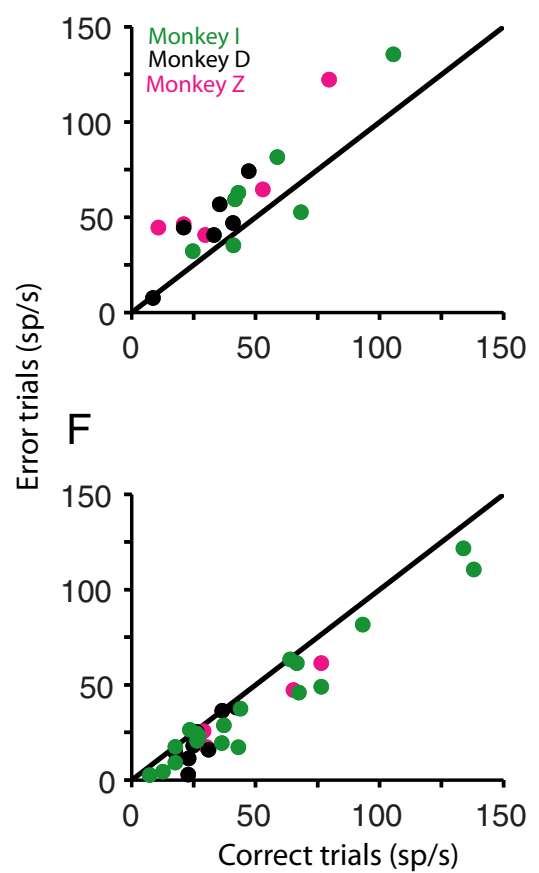

Figure 6. Target and distractor responses predict saccadic targeting during small-reward error trials. A lower target response predicts saccades away from the target. A higher distractor response on such trials predicts a distractor-directed saccade. $\boldsymbol{A}$, Schematic depicting distractor-directed error trials. $\boldsymbol{B}$, Population average PSTH shows larger distractor responses on distractor-directed error trials (red) than on correct trials (blue). C, Scatter plot of each neuron's response to the distractor during correct trials (abscissa) and distractor-directed error trials (ordinate). Paired $t$ test: $p=0.0003$, $n=18$ neurons. $\boldsymbol{D}$, Schematic depicting small-reward error trials where monkey saccades away from the target. $\boldsymbol{E}$, Population average PSTH shows reduced target responses on these error trials (red) compared to correct trials (blue). Black crosses are as in Figure $2 A$. F, Scatter plot of each neuron's response to the target ( -400 to $300 \mathrm{~ms}$ relative to distractor onset, gray bar in $E$ ) during correct trials (abscissa) and error trials (ordinate). Paired t test: $p<0.0001, n=31$ neurons. PSTH bin widths $=25 \mathrm{~ms}$. Only errors made at least $350 \mathrm{~ms}$ after distractor onset included. Figure format for PSTHs and scatter plots as in Figure 2.

get response in our single-target task conflicts with prior claims based on data from game-based choice tasks (Dorris and Glimcher, 2004), but is entirely consistent with reports from various other parts of the brain, including the dorsolateral prefrontal cortex, frontal and supplementary eye fields, the basal ganglia, and the SC (Ikeda and Hikosaka, 2003; McCoy and Platt, 2005; Hikosaka et al., 2006; Watanabe, 2007).

Spatial attention has been shown to suppress distractor responses through center-surround interactions in the nearsurround of V4 neurons (Sundberg et al., 2009) and from locations far outside the RF in V1 neurons, especially at higher task difficulties (Chen et al., 2008). We suggest based on our results that the center-surround interactions measured in V1 reflect long-range suppressive effects established in another cortical area like LIP, especially if enhancement of surround suppression by higher task difficulty is based on the same circuitry as the enhancement of surround suppression by higher reward expectation (Maunsell, 2004).

\section{Surround suppression in priority maps}

Given the ubiquity of surround suppression in the visual system, it is surprising that the existence of surround suppression in LIP has been controversial (Churchland et al., 2008). Data from other putative priority map areas in the brain has also been inconclusive. Data from FEF during a visual search task indicate only spatially local suppressive effects in less than one-third of neurons that extend on average up to $4-6^{\circ}$ outside the RF at $10^{\circ}$ eccentricity (Schall et al., 1995, 2004). In the SC, distractors flashed at locations distant from the excitatory RF evoke shortlatency suppression of pretarget activity in a gap-saccade task in some neurons (Dorris et al., 2007). In dorsolateral prefrontal cortex (DLPFC), a memory-guided saccade plan has been shown to slightly suppress baseline neuronal responses opposite to the saccade goal (Constantinidis et al., 2002). Our characterization of surround suppression goes well beyond these prior descriptions by unequivocally demonstrating the spatially wide-ranging, strong, and ubiquitous influence of surround suppression of LIP responses and by illustrating several important and novel properties that have not been previously reported from other areas (using tasks that were not explicitly designed to study surround suppression).

The suppressive effects we have measured in LIP may not necessarily be mediated by direct suppressive effects upon LIP neurons, and could instead reflect suppressive interactions in other areas that are then relayed to LIP. This is of course also true for every other response property measured from LIP neurons to date: there is not a single known response property of LIP neurons that is known to be definitively established within LIP itself. However, we do know that inhibitory inputs exert a strong influence on LIP neurons, since the majority of LIP neurons respond with dramatically increased firing after iontophoretic injection of bicuculline hydrochloride, a $\mathrm{GABA}_{\mathrm{A}}$ receptor antagonist, and decreased responses after injection of GABA (Zhang et al., 2007). Because LIP surround suppression can be maintained by a memory-guided saccade plan, it is likely to emerge from one or more "priority-map" brain areas active during the delay period of the memory-guided saccade task like LIP itself (Sereno and Amador, 2006) FEF, DLPFC, and SC. These areas also contain neurons whose activity is enhanced when a larger reward is expected following a saccade into the neuron's RF (Watanabe, 2007), in turn 
leading to enhanced suppression of neurons representing competing locations. The reward-cue signal that mediates the effects of motivation on surround suppression could emerge from a range of areas, including the amygdala, striatum, and the perirhinal, entorhinal, orbitofrontal, and insular cortices (Salzman et al., 2005; Simmons et al., 2007).

\section{Implications of LIP surround suppression for behavior}

LIP represents a priority map whose peak can be used by the visual system for the allocation of visual attention (Bisley and Goldberg, 2003a) and the oculomotor system for the generation of saccades when saccades are appropriate (Ipata et al., 2006). Network models of priority maps often posit that global inhibitory interactions enable the emergence of a peak of activity on the priority map (Koch and Ullman, 1985; Itti and Koch, 2001) and are critical for the maintenance of stable, localized persistent activity that is resistant to perturbation from external distractors (Constantinidis and Wang, 2004). Mutually suppressive pushpull interactions in LIP have also been postulated to be critical for the programming of sequential saccades (Xing and Andersen, 2000).

Studies of perceptual decision making in LIP often model the decision process as a race to a threshold between competing alternatives (Gold and Shadlen, 2007). Our data supports models of perceptual decision making that incorporate mutual inhibition between competing choices (Usher and McClelland, 2001) and argues against models of the decision process as independent random walks toward a threshold. Specifically, our finding that surround suppression can strongly suppress visually driven LIP responses suggests that surround suppression could explain the recently reported large reductions in LIP responses to stimulus onset in a four-choice task compared to a two-choice task (Churchland et al., 2008). Surround suppression of LIP responses also provides a mechanism for the baseline resetting that is part of models for evidence accumulation toward a saccadic decision in LIP (Seung et al., 2000; Roitman and Shadlen, 2002). The reward modulation of surround suppression immediately provides a possible mechanism for the global computations of normalized reward value that have been hypothesized to underlie saccadic choice decisions in LIP (Sugrue et al., 2004).

Converging physiological evidence indicates that the firingrates of neurons in priority maps in FEF, LIP, and SC are tightly linked to attentional allocation (Bisley and Goldberg, 2003b; Armstrong et al., 2009). Our findings provide a mechanism by which spatial locations can compete with each other for attentional priority: the higher firing-rates associated with LIP neurons representing a particular location (in this case, the saccade goal) lead to lower firing rates in neurons representing other competing locations. Consistent with the tight linkage between LIP activity and spatial attention, this mechanism correlates with a reduction of attentional allocation to the RF when the RF location competes with the saccade goal outside the RF for attentional priority.

It could be argued that attention is the cause of the reduced responses to the distractor in LIP in the presence of the saccade plan to a competing spatial location. This argument posits that the distractor response is lower in the presence of a saccade plan because the distractor is less likely to capture attention than the distractor in the no-saccade control condition. However, attention is a psychophysical construct and cannot cause neural effects. Instead, it is the neural implementation of attention that must cause the measured effects in LIP, not attention itself. Therefore, this argument is identical to one discussed above where the suppressive effects measured in LIP reflect suppressive interactions in other areas that are then relayed to LIP. The surround suppression we measure in LIP, whether it is implemented in LIP or elsewhere, provides a mechanism that could underlie the ability of focused attention to modulate attentional capture by an abruptly appearing irrelevant stimulus in human subjects (Egeth and Yantis, 1997). In keeping with the broad extent of the suppressive surrounds we find in LIP, a human patient with bilateral parietal lesions had difficulty filtering distractors even over large distances (Friedman-Hill et al., 2003). The increased suppression of distractor responses in LIP when a higher reward is cued provides a physiological basis for possible improvements in attentional focusing and distractor suppression as a function of the cognitive demands of the task.

\section{References}

Alitto HJ, Usrey WM (2008) Origin and dynamics of extraclassical suppression in the lateral geniculate nucleus of the macaque monkey. Neuron 57:135-146.

Allman J, Miezin F, McGuinness E (1985) Stimulus specific responses from beyond the classical receptive field: neurophysiological mechanisms for local-global comparisons in visual neurons. Annu Rev Neurosci 8:407-430.

Angelucci A, Bressloff PC (2006) Contribution of feedforward, lateral and feedback connections to the classical receptive field center and extraclassical receptive field surround of primate V1 neurons. Prog Brain Res 154:93-120.

Armstrong KM, Chang MH, Moore T (2009) Selection and maintenance of spatial information by frontal eye field neurons. J Neurosci 29: 15621-15629.

Balan PF, Oristaglio J, Schneider DM, Gottlieb J (2008) Neuronal correlates of the set-size effect in monkey lateral intraparietal area. PLoS Biol 6:e158.

Bisley JW, Goldberg ME (2003a) Neuronal activity in the lateral intraparietal area and spatial attention. Science 299:81-86.

Bisley JW, Goldberg ME (2003b) The role of the parietal cortex in the neural processing of saccadic eye movements. Adv Neurol 93:141-157.

Chen Y, Martinez-Conde S, Macknik SL, Bereshpolova Y, Swadlow HA, Alonso JM (2008) Task difficulty modulates the activity of specific neuronal populations in primary visual cortex. Nat Neurosci 11:974-982.

Churchland AK, Kiani R, Shadlen MN (2008) Decision-making with multiple alternatives. Nat Neurosci 11:693-702.

Constantinidis C, Wang XJ (2004) A neural circuit basis for spatial working memory. Neuroscientist 10:553-565.

Constantinidis C, Williams GV, Goldman-Rakic PS (2002) A role for inhibition in shaping the temporal flow of information in prefrontal cortex. Nat Neurosci 5:175-180.

Deco G, Pollatos O, Zihl J (2002) The time course of selective visual attention: theory and experiments. Vision Res 42:2925-2945.

Desimone R, Schein SJ, Moran J, Ungerleider LG (1985) Contour, color and shape analysis beyond the striate cortex. Vision Res 25:441-452.

Desimone R, Moran J, Schein SJ, Mishkin M (1993) A role for the corpus callosum in visual area V4 of the macaque. Vis Neurosci 10:159-171.

Dorris MC, Glimcher PW (2004) Activity in posterior parietal cortex is correlated with the relative subjective desirability of action. Neuron 44:365-378.

Dorris MC, Olivier E, Munoz DP (2007) Competitive integration of visual and preparatory signals in the superior colliculus during saccadic programming. J Neurosci 27:5053-5062.

Egeth HE, Yantis S (1997) Visual attention: control, representation, and time course. Annu Rev Psychol 48:269-297.

Eifuku S, Wurtz RH (1998) Response to motion in extrastriate area MSTl: center-surround interactions. J Neurophysiol 80:282-296.

Fecteau JH, Munoz DP (2006) Salience, relevance, and firing: a priority map for target selection. Trends Cogn Sci 10:382-390.

Friedman-Hill SR, Robertson LC, Desimone R, Ungerleider LG (2003) Posterior parietal cortex and the filtering of distractors. Proc Natl Acad Sci U S A 100:4263-4268.

Gnadt JW, Andersen RA (1988) Memory related motor planning activity in posterior parietal cortex of macaque. Exp Brain Res 70:216-220. 
Gold JI, Shadlen MN (2000) Representation of a perceptual decision in developing oculomotor commands. Nature 404:390-394.

Gold JI, Shadlen MN (2007) The neural basis of decision making. Annu Rev Neurosci 30:535-574.

Goldberg ME, Bisley JW, Powell KD, Gottlieb J (2006) Saccades, salience and attention: the role of the lateral intraparietal area in visual behavior. Prog Brain Res 155:157-175.

Hikosaka O, Nakamura K, Nakahara H (2006) Basal ganglia orient eyes to reward. J Neurophysiol 95:567-584.

Ikeda T, Hikosaka O (2003) Reward-dependent gain and bias of visual responses in primate superior colliculus. Neuron 39:693-700.

Ipata AE, Gee AL, Goldberg ME, Bisley JW (2006) Activity in the lateral intraparietal area predicts the goal and latency of saccades in a freeviewing visual search task. J Neurosci 26:3656-3661.

Itti L, Koch C (2000) A saliency-based search mechanism for overt and covert shifts of visual attention. Vision Res 40:1489-1506.

Itti L, Koch C (2001) Computational modelling of visual attention. Nat Rev Neurosci 2:194-203.

Keller EL, McPeek RM (2002) Neural discharge in the superior colliculus during target search paradigms. Ann N Y Acad Sci 956:130-142.

Koch C, Ullman S (1985) Shifts in selective visual attention: towards the underlying neural circuitry. Hum Neurobiol 4:219-227.

Maunsell JH (2004) Neuronal representations of cognitive state: reward or attention? Trends Cogn Sci 8:261-265.

McCoy AN, Platt ML (2005) Expectations and outcomes: decision-making in the primate brain. J Comp Physiol A Neuroethol Sens Neural Behav Physiol 191:201-211.

Orban GA (2008) Higher order visual processing in macaque extrastriate cortex. Physiol Rev 88:59-89.

Peck CJ, Jangraw DC, Suzuki M, Efem R, Gottlieb J (2009) Reward modulates attention independently of action value in posterior parietal cortex. J Neurosci 29:11182-11191.

Roesch MR, Olson CR (2003) Impact of expected reward on neuronal activity in prefrontal cortex, frontal and supplementary eye fields and premotor cortex. J Neurophysiol 90:1766-1789.

Roitman JD, Shadlen MN (2002) Response of neurons in the lateral intraparietal area during a combined visual discrimination reaction time task. J Neurosci 22:9475-9489.

Salzman CD, Belova MA, Paton JJ (2005) Beetles, boxes and brain cells: neural mechanisms underlying valuation and learning. Curr Opin Neurobiol 15:721-729.
Schall JD (1995) Neural basis of saccade target selection. Rev Neurosci 6:63-85.

Schall JD, Hanes DP, Thompson KG, King DJ (1995) Saccade target selection in frontal eye field of macaque. I. Visual and premovement activation. J Neurosci 15:6905-6918.

Schall JD, Sato TR, Thompson KG, Vaughn AA, Juan CH (2004) Effects of search efficiency on surround suppression during visual selection in frontal eye field. J Neurophysiol 91:2765-2769.

Serences JT, Yantis S (2007) Spatially selective representations of voluntary and stimulus-driven attentional priority in human occipital, parietal, and frontal cortex. Cereb Cortex 17:284-293.

Sereno AB, Amador SC (2006) Attention and memory-related responses of neurons in the lateral intraparietal area during spatial and shape-delayed match-to-sample tasks. J Neurophysiol 95:1078-1098.

Seung HS, Lee DD, Reis BY, Tank DW (2000) Stability of the memory of eye position in a recurrent network of conductance-based model neurons. Neuron 26:259-271.

Simmons JM, Ravel S, Shidara M, Richmond BJ (2007) A comparison of reward-contingent neuronal activity in monkey orbitofrontal cortex and ventral striatum: guiding actions toward rewards. Ann N Y Acad Sci 1121:376-394.

Sugrue LP, Corrado GS, Newsome WT (2004) Matching behavior and the representation of value in the parietal cortex. Science 304:1782-1787.

Sundberg KA, Mitchell JF, Reynolds JH (2009) Spatial attention modulates center-surround interactions in macaque visual area v4. Neuron 61:952-963.

Thompson KG, Bichot NP (2005) A visual salience map in the primate frontal eye field. Prog Brain Res 147:251-262.

Usher M, McClelland JL (2001) The time course of perceptual choice: the leaky, competing accumulator model. Psychol Rev 108:550-592.

Watanabe M (2007) Role of anticipated reward in cognitive behavioral control. Curr Opin Neurobiol 17:213-219.

Wong KF, Huk AC, Shadlen MN, Wang XJ (2007) Neural circuit dynamics underlying accumulation of time-varying evidence during perceptual decision making. Front Comput Neurosci 1:6.

Xing J, Andersen RA (2000) Memory activity of LIP neurons for sequential eye movements simulated with neural networks. J Neurophysiol 84:651-665.

Zhang M, Wang X, Goldberg ME (2007) GABAergic inhibitory mechanisms shape the response properties of parietal neurons in the monkey. Soc Neurosci Abstr 33:280.19. 